\title{
Formación en técnicas de primeros auxilios a los estudiantes del bachillerato de la sección nocturna del Instituto Superior José Peralta del Cantón Cañar
}

\author{
Aguaiza Pichazaca Erlinda* y Paguay Muñoz María Augusta \\ Carrera de Enfermería, Universidad Católica de Cuenca \\ Cañar, 030350, Ecuador \\ *eaguaizap@ucacue.edu.ec
}

\begin{abstract}
Resumen
El artículo se trata sobre la Formación en Técnicas de Primeros Auxilios se realiza con el propósito de fundamentar conocimientos y utilización de técnicas en los estudiantes de Bachillerato de Sección Nocturna del Instituto "José Peralta", el objetivo general es favorecer la adquisición de conocimientos en los alumnos acerca de primeros auxilios, para fundamentar teóricamente el trabajo de investigación se consideró las siguientes fuentes de la bibliografía como: Manuales, libros, enciclopedia, internet etc. Como técnica e instrumentos utilizados es la encuestas donde nos da a conocer que el $70 \%$ de los estudiantes no tiene conocimiento sobre primeros auxilios, el $30 \%$ sí, la población de estudio comprende el $56 \%$ es de sexo femenino, el $44 \%$ de sexo masculino, de acuerdo con los resultados obtenidos podemos darnos cuenta que son estudiantes de 19 a 23 años de edad. En la metodología de la investigación, se emplearon métodos y técnicas que permitieron la recopilación de la información y el análisis de la misma; El presente trabajo fue desarrollado con una muestra de la población total.
\end{abstract}

Palabras clave: Primeros auxilios, técnica, instructivo, tratamiento, actuación, inmediata, conocimiento, prevención.

\begin{abstract}
The article deals with the training in first aid techniques, it is developed in order to set knowledgeand use techniques in high school students at the Eveningsection of the "Jose Peralta" Institute, the general objective is to promote the acquisition of knowledge in students regarding first aid, in order to theoretically support this research the following literature sources were considered: Guidebooks, books, encyclopedia, internet etc. The survey is the technique and instrument used, it presents that $70 \%$ of the students do not have any knowledge regarding first aid, $30 \%$ do have, 56\% of the population is female, 44\% male, according to the results, and we can realize that they are students between 19 and 23 years old. Methods and techniques that allowed the collection of the information and its analysis were used in the methodology of this research; thisresearch work was developed with a sample of the total population.
\end{abstract}

Key words: First aid, technical, instructional, treatment, acting, immediate, knowledge, prevention.

\section{Introducción}

Primeros auxilios y entornos educativos proyecto de convergencia realizado en España por la Sociedad Española de Enfermería de Urgencias y Emergencia, también en la ESCUELA NACIONAL DE SALUD PUBLICA CIUDAD DE LA HABANA SE REALIZO UN Proyecto de "Enseñanza de los primeros auxilios a escolares de 4to a 9no grados" demostrando así que es un tema de interés mundial ya que si impartimos conocimientos sobre el tema a estas edades lograremos bajar el índice de muertes gracias a que sabrán actuar de manera correcta e incluso podrán compartir sus conocimientos en los hogares con su familia ${ }^{1}$.

Según el INSTITUTO NACIONAL DE ESTADISTICAS Y CENSO (INEC) encuesta realizada en los años
2005 a 2013 las estadísticas de accidentes a nivel Nacional generan los siguientes datos tomando en cuenta esta información podemos ver que la provincia del Cañar tiene un número considerable de casos con respecto a los demás provincias que tienen mayor población, por lo que es importante el desarrollo de este tipo de trabajo que se enfoca en capacitar a la población para que sepan cómo actuar ante algunas de estas situaciones ${ }^{2}$.

En los últimos tiempos el problema que viene presentando dentro del contexto social no existe capacitación e información acerca del tema de primeros auxilios especialmente en las instituciones educativas del cantón cañar, tanto en los estudiantes como en los docentes no poseen suficientes conocimientos para enfrentar en una situación 
Tabla 1. Datos estadísticos de accidentes a nivel nacional

\begin{tabular}{|c|c|c|c|c|c|c|c|c|c|c|}
\hline \multirow{3}{*}{ PROVINCIAS } & \multicolumn{10}{|c|}{ VICTIMAS } \\
\hline & \multicolumn{3}{|c|}{ MUERTOS } & \multicolumn{3}{|c|}{ HERIDOS } & \multicolumn{3}{|c|}{ TRAUMATTCOS } & \multirow[b]{2}{*}{ T. } \\
\hline & Total & $H$ & M & $T$ & $\mathrm{H}$ & M & $T$ & $\mathrm{H}$ & M & \\
\hline & & & & & & & & & & \\
\hline AZUAY & 81 & 61 & 20 & 444 & 273 & 171 & 12 & 10 & 2 & 537 \\
\hline BOLIVAR & 21 & 13 & 8 & 59 & 42 & 17 & 3 & 0 & 3 & 83 \\
\hline CANAR & 20 & 15 & 5 & 256 & 148 & 108 & 0 & 0 & 0 & 276 \\
\hline CARCHI & 36 & 34 & 2 & 151 & 94 & 57 & 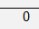 & 0 & 0 & 187 \\
\hline COTOPAXI & 100 & 73 & 27 & 402 & 249 & 153 & 0 & 0 & 0 & 502 \\
\hline CHIMBORAZO & 52 & 35 & 17 & 343 & 213 & 130 & 0 & 0 & 0 & 395 \\
\hline EL ORO & 48 & 38 & 10 & 217 & 162 & 55 & 10 & 6 & 4 & 275 \\
\hline ESMERALDAS & 43 & 26 & 17 & 327 & 234 & 93 & 5 & 3 & 2 & 375 \\
\hline GUAYAS & 242 & 165 & 73 & 1.73 & 1.115 & 617 & 126 & 101 & 25 & 2100 \\
\hline IMBABURA & 74 & 53 & 21 & 368 & 233 & 135 & 6 & 3 & 3 & 448 \\
\hline LOJA & 32 & 24 & 8 & 107 & 79 & 28 & 17 & 13 & 4 & 156 \\
\hline LOS RIOS & 87 & 73 & 14 & 454 & 354 & 100 & 0 & 0 & 0 & 541 \\
\hline MANABI & 83 & 66 & 17 & 571 & 403 & 168 & 18 & 9 & 9 & 672 \\
\hline $\begin{array}{l}\text { MORONA } \\
\text { SANTAGO }\end{array}$ & 1 & 1 & 0 & 91 & 57 & 34 & 0 & 0 & 0 & 92 \\
\hline NAPOPAST & 13 & 11 & 2 & 50 & 40 & 10 & 0 & 0 & 0 & 63 \\
\hline PASTAZA & 3 & 3 & 0 & 55 & 30 & 25 & 1 & 0 & 1 & 59 \\
\hline PICHINCHA & 340 & 240 & 100 & 2.81 & 1.796 & 1.015 & 2 & 1 & 1 & 3.153 \\
\hline TUNGURAHUA & 71 & 55 & 16 & 447 & 264 & 183 & 0 & 0 & 0 & 518 \\
\hline $\begin{array}{l}\text { ZAMORA } \\
\text { CHINCHIPE }\end{array}$ & 10 & 10 & 0 & 54 & 45 & 9 & 0 & 0 & 0 & 64 \\
\hline GALAPAGOS & 2 & 2 & 0 & 18 & 9 & 9 & 0 & 0 & 0 & 20 \\
\hline SUCUMBIOS & 14 & 13 & 1 & 115 & 79 & 36 & 12 & 6 & 6 & 141 \\
\hline ORELLANA & 14 & 13 & 1 & 26 & 21 & 5 & 0 & 0 & 0 & 40 \\
\hline
\end{tabular}

de accidentes.

Siendo primeros auxilios uno de los temas muy importantes dentro de la sociedad ecuatoriana ya que vivimos los momentos muy difíciles en estos últimos tiempos, por tal motivo debemos conocer o saber cómo actuar cuando presente una situación en cualquier momento de la vida.

El objetivo de este trabajo de investigación se enfoca en favorecer la adquisición y utilización de técnicas sobre los Primeros Auxilios y determinar en los estudiantes habilidades y destrezas, elaborar talleres prácticos para fortalecer el conocimiento de cómo actuar frente a un accidente y además diseñar instructivo de Primeros Auxilios para los estudiantes.

\section{Materiales y Métodos}

El tipo de investigación utilizada fue de campo y descriptiva por cuanto facilito establecer más a fondo las informaciones acerca de conocimiento sobre primeros auxilios que tienen los estudiantes del Bachillerato del Instituto "José peralta".

El número total de la población, es de 120 estudiantes del bachillerato de la sección Nocturna del Instituto Superior José Peralta de la ciudad de Cañar.

El tipo de muestra que se tomó en cuenta para la elaboración de la investigación es el probabilístico al azar simple ya que todos los estudiantes tienen la posibilidad de obtener los conocimientos impartidos y se seleccionó al azar considerando a 54 estudiantes del Bachillerato.

Durante el proceso de la investigación se utilizó los diferentes materiales como: Computadora, impresora, papel bond, Flash memory, Cd. Fuentes de consultas como. Internet, libros, Revistas, artículos etc.

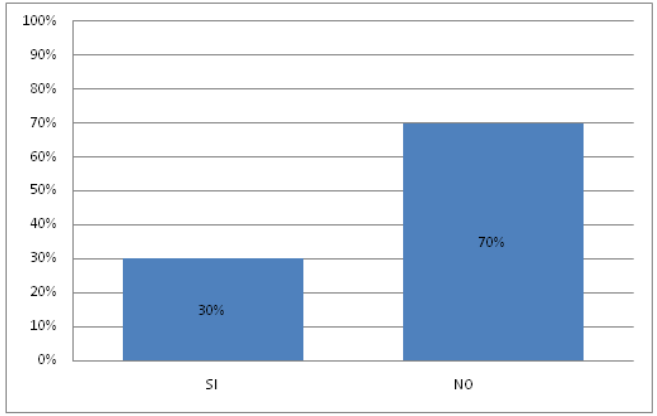

Fig. 1. Grado de conocimientos que tienen los estudiantes sobre primeros auxilios

Técnicas e instrumentos utilizados fue la encuesta con una serie de 13 preguntas, dirigida a los estudiantes del Instituto "José Peralta," una vez obtenida la información de las encuestas aplicadas a los estudiantes se procedió realizar el análisis de datos.

\section{Resultados}

Luego de la aplicación de las encuestas se obtiene los siguientes resultados, el $52 \%$ de los estudiantes tiene entre 19 a 23 años de edad, la mayor parte corresponde al sexo femenino.

El $70 \%$ no tiene conocimiento sobre primeros auxilios, por lo que la elaboración de este trabajo fue de gran importancia para poder brindarles información sobre este tema ya que en algún momento de su vida será necesario, a continuación se demuestra mediante el gráfico

El $39 \%$ de los encuestados desconocen cuál es el objetivo de los primeros auxilios, $32 \%$ cree que es evitar autolesionarse, el $20 \%$ conservar la vida, $9 \%$ llamar al $911,35 \%$ de los estudiantes no pueden actuar frente a una quemadura, fractura, por lo que en el taller también se les indico que hacer y no hacer ante estos casos también como reconocer por lo que se les brindo conocimientos sobre los tipos de fracturas, como reconocerlas y cómo actuar ante cada caso para evitar causar mayor daño, en referente al botiquín el $37 \%$ de los encuestados cree que debe tener un botiquín en la casa, el $33 \%$ en el aula, el $19 \%$ en el auto, $11 \%$ no sabe, ante un parto de urgencia en que tiempo debo ligar el cordón umbilical, el $39 \%$ de los estudiantes desconocen el tiempo para ligar el cordón umbilical, el $26 \%$ cree que en $15 \mathrm{sg}$, el $22 \% 30 \mathrm{sg}, 13 \%$ en 1 minuto, también desconocen las medidas de seguridad tanto para la madre, el niño y el auxiliador, el $100 \%$ cree que sería importante recibir una charla o un taller sobre primeros auxilios en la institución a la que asisten, nos podemos dar cuenta que la mayoría de estudiantes no tiene conocimiento o sabe poco sobre el tema para lo cual se realizó una capacitación y taller a los estudiantes con el fin de proporcionar conocimientos sobre los primeros auxilios. 


\section{Discusión}

El trabajo de investigación fue de tipo descriptivo y de campo, Formación en Técnicas en Primeros Auxilios en el Bachillerato Sección Nocturna, luego de la aplicación de la encuesta se determina que existe un alto porcentaje de estudiantes que no tiene conocimiento sobre primeros auxilios, por lo que la elaboración de este trabajo fue de gran importancia para poder proporcionar conocimientos acerca de cómo actuar frente a una persona accidentado

La mayoría de los estudiantes no saben cuál es el objetivo de los primeros auxilios y las autoridades deberían realizar talleres, cursos de capacitación, elaborar instructivos y otros. Frente a esta realidad que vive la Institución Educativa se ha organizado un Seminario Taller obteniendo resultados positivos, logrando así darse cuenta cual es el objetivo de los primeros auxilios, se les indico que hacer y no hacer ante estos casos también se fomentó conocimientos sobre los tipos de fracturas, como reconocerlas y cómo actuar ante cada caso para evitar causar mayor daño.

Es muy importante que en todos los vehículos, hogares e instituciones disponer de un botiquín con todos los elementos necesarios ya que los accidentes no solo ocasionan en las calles, por ende este trabajo de investigación se orienta en mejorar los conocimientos básicos sobre la Formación en Técnicas de Primeros Auxilios en los estudiantes de Bachillerato Sección Nocturna del Instituto "José Peralta" de la ciudad de Cañar.

\section{Conclusiones}

- Mediante los resultados obtenidos del objetivo uno se puede concluir que la mayoría de los estudiantes no tienen conocimientos acerca de primeros auxilios para lo cual se ha realizado un seminario taller con los estudiantes.

- En relación con las encuestas aplicadas el colegio no cuenta con un instructivo de primeros auxilios, por tal motivo se ha diseñado el instructivo educativo.

- Durante el taller los estudiantes han demostrado interés y responden correctamente las preguntas de evaluación.

- El tema de investigación de primeros auxilios tuvo gran acogida por todos los docentes y estudiantes del Instituto "José Peralta".

\section{Recomendaciones}

- Se recomienda a las autoridades de la institución continuar organizando capacitaciones y talleres educativos de primeros auxilios y así mejorar los conocimientos.

- Proporcionar de un manual de primeros auxilios a todos los docentes de los colegios del Cantón Cañar para que los docentes personas tengan conocimientos para actuar ante una emergencia y de esta manera evitar daños.
- Realizar simulacros y charlas con los estudiantes sobre este tema ya que es de gran importancia y nos servirá toda la vida.

- Adquirir de un botiquín en cada aula para casos de emergencia y actuar de manera adecuada.

- Se recomienda a todos los docentes y estudiantes aplicar decentemente el instructivo de primeros auxilios.

\section{Abreviaturas}

- RCP: Reanimación Cardio Pulmonar item ABC

- A abrir vía aérea y control de cervicales

- B ventilación

○ C: circulación y control de hemorragia

- $\mathrm{ABC}$

- $\mathrm{S}$ signos y síntomas: qué veo y qué me dice el paciente que siente

- A alergias: a medicamentos o a alguna otra cosa

- M medicamentos: qué medicamentos consume

- P padecimientos: qué enfermedades padece

- L ultima ingesta: qué fue lo último que consumió de alimentos especificándolos y líquidos bebidos.

- E eventos relacionados al trauma o enfermedad:

\section{Agradecimientos}

Agradezco a mi Dios por haberme permitido realizar esta gran tarea, dándome fuerza para continua en la lucha a favor de aquellas personas que necesitan para el alivio de su dolor.

Se reconoce a las personas que participaron directa e indirectamente en la investigación, tales como personal que laboran en el Instituto "José Peralta", otras en general.

Agradezco de manera especial a las autoridades de la Universidad Católica de Cuenca Extensión Cañar por abrir las puertas para poder ser parte de la Institución de Educación Superior.

\section{Referencias}

1. Rodríguez AO. Especialista en Salud Publica en Emergencias y Desastres. SE; 2011.

2. Navarrete P SIJ. Manual de Soporte Vital Avanzado en Trauma. $4^{\mathrm{a}}$ ed. Plan Nacional de RCP.; 2007.

Recibido: 01 de junio de 2016

Aceptado: 30 de mayo de 2017

Aguaiza Pichazaca E: Licenciada en Enfermería, Coordinadora de Practicas Preprofesionales,Ex-Directora de 
Carrera de Enfermeria, Docente Universitaria, Publicación de Articulo Científico,Magister en Gerencia para el Desarrollo Local

Paguay Muñoz Maria Augusta: Auxiliar de Enfermeria, Licenciada en Enfermería,Mejor Egresada de la Carrera de Enfermería, Cursando Maestria en Master de Especialización en Unidades de Enfermería. Correo electrónico: matus1990@hotmail.com 
